\section{Controversias respecto al uso de estatinas en el síndrome coronario agudo}

\section{Controversies regarding the use of statins in the acute coronary syndrome}

\section{Estimado Editor:}

He leído el artículo de Ruiz Bailen ${ }^{1}$, respecto a las estatinas en la fase aguda del síndrome coronario agudo, y coincido respecto a que falta evidencia clara que respalde su uso. Sin embargo, a pesar de lo que expresa el autor, no podríamos sugerirlos como terapéutica habitual para pacientes con el síndrome hasta que no se diluciden algunas de las hipótesis sobre los mecanismos de acción y efectos pleiotrópicos de las estatinas.

El eje de algunos de los efectos pleiotrópicos de las estatinas es la disminución del mevalonato que es el precursor del colesterol y de compuestos isoprenoides no esteroideos, al lograr la inhibición de la HMG-CoA reductasa ${ }^{2}$.

Tales efectos incluyen varios que resultan beneficiosos y cardioprotectivos, como mejoría en la función endotelial, incremento de la biodisponibilidad de óxido nítrico, y otros como una actividad inmunosupresora paulatina, capacidad de reclutar células progenitoras endoteliales e inhibición de la hipertrofia ventricular ${ }^{3}$.

Sin embargo, al inhibir la síntesis de ácido mevalónico desciende la producción de ubiquinona (o coenzima Q10) que se encuentra en cantidades importantes en el corazón y es un componente esencial de la cadena respiratoria mitocondrial. Las estatinas, al interferir en la síntesis de ubiquinona afectan la función mitocondrial y producen un efecto deletéreo sobre el músculo cardiaco, al disminuir la producción de ATP y desacoplar la fosforilación oxidativa produciendo radicales libres ${ }^{4}$.

Otro efecto no benéfico de las estatinas es la disminución de la selenocisteína-tRNA, que controla la expresión de todas las selenoproteínas. Al ser interrumpida la vía del mevalonato, la selenoproteína-tRNA no puede transformarse en una molécula funcional. Tanto la disminución de la ubiquinona como la de selenoproteínas, estarían vinculadas a la miopatía por estatinas ${ }^{5}$.

Por ello, y a pesar que muchos pacientes con patología coronaria que ingresan a nuestras unidades de cuidados intensivos ya tienen una terapéutica usual con estatinas, debemos ser cautos al prescribir o indicar estos fármacos en pacientes con síndrome coronario agudo, y monitorizar aquellos que ya los consumen. Se debe sugerir fuertemente la realización de ensayos clínicos sobre la incorporación de estatinas en el arsenal terapéutico de un paciente con síndrome coronario agudo, y también se deben apoyar las investigaciones experimentales que nos permitan conocer los aspectos aún no aclarados de estos fármacos.

\section{Bibliografía}

1. Ruiz-Ballen M. Administración de estatinas durante la fase aguda del síndrome coronario agudo. Med Intensiva. 2010;34:56-63.

2. Istvan ES, Deisenhofer J. Structural mechanism for statin inhibition of HMG-CoA reductasa. Science. 2001;292:1160-4.

3. Davignon J. The cardioprotective effects of statins. Curr Atheroscler Rep. 2004;6:27-35.

4. Mortensen SA, Leth A, Agner E, Rohde M. Dose-related decrease of serum coenzyme Q10 during treatment with HMG-CoA reductase inhibitors. Mol Aspects Med. 1997;18:S137-44.

5. Moosmann B, Behl C. Selenoprotein synthesis and dide-effects of statins. Lancet. 2004;363:892-4.

\section{Camps ${ }^{\mathrm{a}, \mathrm{b}}$}

${ }^{a}$ Facultad de Medicina, Universidad Católica de Córdoba, Argentina

bUnidad de Terapia Intensiva, Clínica Privada Modelo

de Río Tercero, Córdoba, Argentina

Correo electrónico: editorfm@gmail.com

\section{Administración precoz de estatinas sobre el síndrome coronario agudo}

\section{Early administration of statins in acute coronary syndrome}

\section{Sr. Editor:}

En respuesta a la carta del Dr. Camps sobre el «uso precoz de las estatinas en el síndrome coronario agudo (SCA)», coincido, y así lo expongo en el texto original ${ }^{1}$, en la existencia de controversia en la administración precoz de estatinas, pues existen muchos estudios con gran heterogeneidad, aunque la lectura de estos muestra que la mayoría de ellos coincide en encontrar efectos beneficiosos en la administración de estatinas sobre el SCA, beneficio que se podría traducir en una disminución de la mortalidad precoz.
Molyneux ha demostrado en un reciente estudio que el nivel elevado de coenzima Q10 se asocia con una disminución de la mortalidad ante la insuficiencia cardiaca crónica ${ }^{2}$. Las estatinas disminuyen esta coenzima Q10, pero también disminuyen claramente la mortalidad en la enfermedad cardiovascular, lo que podría ser paradójico. No obstante, debemos recordar que esta coenzima Q10 es «una más» de las múltiples vías que se modifican, con la administración de estatinas, en la vía del colesterol. A pesar de que uno de los múltiples efectos deletéreos de las estatinas sean los mencionados por el Dr. Camps, también se conocen múltiples mecanismos bioquímicos por inhibición de «metabolitos intermedios», que claramente tienen efectos deletéreos al alterar el sistema inflamatorio, procoagulante, o la neovascularización, entre otros ${ }^{1}$. En cualquier caso, el resultado medible final es la supervivencia, y este objetivo final parece que es mejor con la administración de estatinas.

Esta carta podría hacer una pregunta aun más interesante: ¿qué tiene más valor, una decisión basada en fisiopatología o una evidencia? Obviamente, ante una débil evidencia 
científica, debemos respetar los conocimientos fisiopatológicos (aunque debemos recordar que la fisiopatología también nos guarda muchos secretos, y estos a veces se interrogan tras los hallazgos encontrados en ensayos clínicos). De igual modo, entiendo que lo ideal sería un ensayo clínico dirigido a la evaluación de la administración de estatinas durante la fase precoz, pero ¿es posible? ¿Cuál sería la muestra requerida? ¿Estarían dispuestas las empresas farmacéuticas implicadas en la situación actual, cuando ya está sentada su indicación a la salida del hospital? Estoy muy de acuerdo con el Dr. Camps, en cuanto a ser cautos para lograr el mayor beneficio de los pacientes. Pero ¿es ser prudente y beneficioso no administrar estatinas, o, lo que podría ser peor, retirar las estatinas durante la fase aguda a los pacientes que ya la tomaban, efecto último que parece ser inductor de mayor morbimortalidad ${ }^{3}$ ? No podemos ni debemos limitar la administración de un fármaco, aunque desconozcamos sus completas vías de acción, si conocemos que el resultado final es una disminución de la morbimortalidad. ¿Acaso conocemos a la perfección todos los mecanismos de los agentes terapéuticos utilizados en la actualidad?

Con respecto a la evidencia actual sobre este tema, considero que es adecuado recomendar la administración de estatinas, de forma precoz, en los pacientes con infarto agudo de miocardio. En el caso del SCA sin elevación del segmento
ST, el grupo de trabajo de la SEMICYUC considera adecuada la administración precoz de estatinas en las primeras $24 \mathrm{~h}^{4}$.

\section{Bibliografía}

1. Ruiz-Bailén M. Administración de estatinas durante la fase aguda del síndrome coronario agudo. Med Intensiva. 2010;34:56-63.

2. Molyneux SL, Florkowski CM, George PM, Pilbrow AP, Frampton CM, Lever M, et al. Coenzyme Q10: An independent predictor of mortality in chronic heart failure. J Am Coll Cardiol. 2008;52:1435-41.

3. Ruiz-Bailén $M$. Efecto de la retirada de las estatinas durante el ingreso en unidades de Medicina Intensiva. Med Intensiva. 2010;34(4):268-72.

4. Civeira Murillo E, Del Nogal Sáez F, Álvarez Ruiz AP, Ferrero Zorita J, Alcántara AG, Aguado $\mathrm{GH}$, et al. Grupo de Trabajo de Cuidados Intensivos Cardiológicos y RCP. Recomendaciones para el diagnóstico y tratamiento del síndrome coronario agudo sin elevación del segmento ST. Med Intensiva. 2010;34:22-45.

\section{Ruiz Bailén}

Servicio de Cuidados Críticos y Urgencias, Unidad de Medicina Intensiva, Complejo Hospitalario de Jaén, Universidad de Jaén, Jaén, España

Correo electrónico: ruizbailen@telefonica.net

\section{¿Influyen los antecedentes de consumo de sustancias adictivas en la necesidad de sedación del paciente crítico?}

\section{Does a history of substance abuse influence sedation requirements in critically-ill patients?}

\section{Sr. Director:}

El fenómeno de la adicción a sustancias de abuso ha alcanzado en los últimos 30 años una extraordinaria importancia, por sus consecuencias sociales y sanitarias ${ }^{1}$. Las sustancias psicoactivas más utilizadas por la población española son el alcohol y el tabaco (entre las drogas legales) y el cannabis, la cocaína y el éxtasis (entre las drogas ilegales). Este consumo puede inducir, por un lado, fenómenos de tolerancia y, por otro, inducción enzimática hepática, que podrían repercutir en las necesidades de sedoanalgesia cuando estos pacientes requieren ingreso en una unidad de cuidados intensivos $(\mathrm{UCI})^{2}$. De hecho, el personal que trabaja en una $\mathrm{UCl}$ tiene la sensación de que cuando ingresan consumidores regulares de drogas de abuso, requieren dosis más altas de fármacos para lograr una sedación y analgesia adecuada.

Para verificar si esta percepción es cierta, se diseñó un estudio prospectivo de 10 meses de duración, realizado en la $\mathrm{UCl}$-quirúrgica de un hospital de alta tecnología y aprobado por el comité de ética y de investigación del centro. Todos los pacientes que precisaron analgesia o sedación durante las primeras $72 \mathrm{~h}$ de su ingreso se dividieron en 2 grupos. El grupo । incluyó pacientes traumáticos, politraumáticos o postoperatorios de cirugía general, con antecedentes recogidos a través de una anamnesis dirigida al paciente o a un familiar próximo del consumo de sustancias adictivas (tabaco, alcohol etílico, heroína, cocaína, anfetaminas o cannabis). El grupo ॥ estaba formado por pacientes con los mismos criterios del grupo ।, pero sin antecedentes del citado consumo. Se evaluaron las necesidades cualitativas y cuantitativas de midazolam, diacepam, propofol, clometiazol, clotiapina, morfina, remifentanilo, metadona, clonidina, paracetamol, ketorolaco y dexketoprofeno, por ser los fármacos más utilizados con finalidad sedoanalgésica en nuestra unidad.

Se incluyeron 54 pacientes, de los cuales 30 (55,6\%) se incorporaron en el grupo । y $24(44,4 \%)$ en el grupo I. La media de edad fue de 49,9 años, el 25,9\% fueron mujeres, el 72,2\% fueron hombres y un transexual, sin observarse diferencias significativas entre ambos grupos. En el grupo de consumidores, las sustancias de abuso más utilizadas fueron el tabaco $(29,6 \%)$, el alcohol etílico $(20,4 \%)$, la cocaína $(9,3 \%)$ y el cannabis $(3,7 \%)$. El análisis estadístico de las necesidades cualitativas y cuantitativas de los fármacos utilizados con finalidad de analgesia o de sedación no mostró ninguna diferencia significativa entre uno $u$ otro grupo. La estancia media en la $\mathrm{UCl}$ del grupo consumidor fue de 12,6 días y de 19,9 días para el grupo no consumidor, con una mortalidad global del $9,3 \%$, sin observarse tampoco diferencias entre ambos grupos.

Este estudio tiene diversas limitaciones, entre éstas el reducido número de casos recogidos, el no haber estratifi- 